\title{
Coexistence Curve of Dilute Polymer Solution in a Mixed Solvent Having Critical Demixing Point
}

\author{
Masako TaKada, Koji OKano, ${ }^{*}$ and Kimio Kurita \\ College of Science and Technology, Nihon University, \\ Tokyo 101, Japan \\ * Faculty of Education, Shinshu University, \\ Nagano 380, Japan
}

(Received December 25, 1992)

\begin{abstract}
The coexistence curves of dilute solutions of Poly(vinyl methyl ether) (PVME) in a solvent mixture methanol/cyclohexane which has a critical demixing point were measured. We found that in the methanol-rich region the presence of a small amount of PVME does not cause any appreciable change in the phase boundary of binary methanol/cyclohexane mixture, whereas in the cyclohexane-rich region it gives rise to a marked shift of phase boundary toward higher temperature region. It is shown that this characteristic phase behavior can be well explained qualitatively on the basis of Flory-Huggins solution theory, together with the interaction parameters deduced from small angle neutron and X-ray scattering experiments, as due to the smallness of the interaction parameter, $\chi_{13}$, between methanol and PVME. The smallness of $\chi_{13}$ can be interpreted as a manifestation of the association of methanol molecules.

KEY WORDS Coexistence Curve / Small-Angle Neutron Scattering / Small-Angle X-Ray Scattering / Spinodal Calculation / Poly(vinyl methyl ether) / Methanol / Cyclohexane / Hydrogen-Bonding /
\end{abstract}

Nearly two decades ago de Gennes argued that a polymer chain dissolved in a solvent mixture having a critical demixing point must contract in the vicinity of the critical point. ${ }^{1}$ This gave rise to a series of subsequent experimental $^{2-4}$ and theoretical ${ }^{5}$ studies, which revealed that the polymer chain does expand at the critical point of the solvent mixture contrary to the de Gennes' prediction.

The present study was motivated by these studies, but our purpose is less ambitious; we would like to discuss the phase diagram of ternary solutions: a system consists of a solvent mixture having a critical demixing point and a small amount of added polymers. Dondos and Izumi already studied experimentally the phase behavior of such ternary systems for several combinations of solvents and polymers. ${ }^{2-4}$ However, in all these systems which they studied the polymer is insoluble in one com- ponent of the solvent mixture; these situations are inconvenient for theoretical analysis.

We therefore studied the phase diagram of a ternary solution: methanol/cyclohexane/poly(vinyl methyl ether), in which poly(vinyl methyl ether)(PVME) is soluble both in methanol and in cyclohexane. We measured the phase diagram which is described in the section of MEASUREMENT OF COEXISTENCE CURVE.

In order to analyze theoretically the observed characteristic phase behavior of the system we need a set of binary interaction parameters $\chi_{12}$, $\chi_{13}$, and $\chi_{23}$ where components 1,2 , and 3 stand respectively for methanol, cyclohexane, and PVME. We could not find the reported values of $\chi_{13}$ and $\chi_{23}$ in literature, so that we determined $\chi_{13}$ via binary cluster integral $B_{1}$ deduced from the small angle neutron scattering (SANS) experiments of semidilute solutions of methanol/PVME system. Whereas 
we determined $\chi_{23}$ by measuring the temperature at which $B_{1}$ vanishes; the temperature dependence of $B_{1}$ was obtained by the small angle X-ray scattering (SAXS) measurements of semidilute solutions of cyclohexane/PVME. These small angle scattering experiments are described in the section of SANS AND SAXS MEASUREMENTS.

In the section of SPINODAL CALCULATION we will show that observed phase behavior can well be explained at least qualitatively by a spinodal calculation by using the values of interaction parameters determined in the section of SANS AND SAXS MEASUREMENTS.

In the final section we summarize our conclusion.

\section{MEASUREMENT OF COEXISTENCE CURVE}

\section{Samples}

PVME obtained from Scientific Polymer Products Co. was freeze-dried and stored in a vacuum box. The PVME was characterized as $M_{n}=4.65 \times 10^{4}$ with $M_{w} / M_{n} \leqq 2.12$ (nominal values). Guaranteed reagent grade methanol and cyclohexane were purchased from Kanto Chemical Co., Inc. and used without further purification.

\section{Method}

Phase separations were carried out in flame sealed tubes (inner diameter $8 \mathrm{~mm}$ ) containing approximately 3 or $4 \mathrm{ml}$ of solutions of different concentrations. The tubes were immersed in a temperature controlled bath $\left( \pm 0.02^{\circ} \mathrm{C}\right)$. The onset of demixing was determined visually by slow (less than $0.02^{\circ} \mathrm{Cmin}^{-1}$ ) cooling of the homogeneous solutions. After thermal equilibration at the temperature of the onset of demixing, the samples were left in the bath undisturbed until phase separation was completed (normally within a few hours). The reproducibility amounted to within $\pm 0.1^{\circ} \mathrm{C}$.

\section{Results}

The coexistence curves of methanol/cyclohexane/PVME systems at constant PVME concentrations thus determined together with that of binary methanol/cyclohexane system ${ }^{6}$ are shown in Figure 1. The volume fraction of cyclohexane in Figure 1 is expressed by $u_{2}$ $=\phi_{2} /\left(\phi_{1}+\phi_{2}\right)$, where $\phi_{1}$ and $\phi_{2}$ are respectively the volume fractions of methanol and cyclohexane in the ternary system. Due to the difficulty of determining the accurate volume fraction of PVME, $\phi_{3}$, the concentration of PVME, $c$, is represented in $\mathrm{g} \mathrm{cm}^{-3}$ in Figure 1.

We see from Figure 1 that within the accuracy of experiment the coexistence curve of methanol/cyclohexane/PVME coincides with that of methanol/cyclohexane in the region of $u_{2}$ lower than about 0.6. In the range $0.6<u_{2}<0.93$, with increasing volume fraction of cyclohexane the demixing temperature increases drastically, then decreases rapidly above $u_{2}=0.93$.

The critical volume fraction of binary methanol/cyclohexane system as expressed by

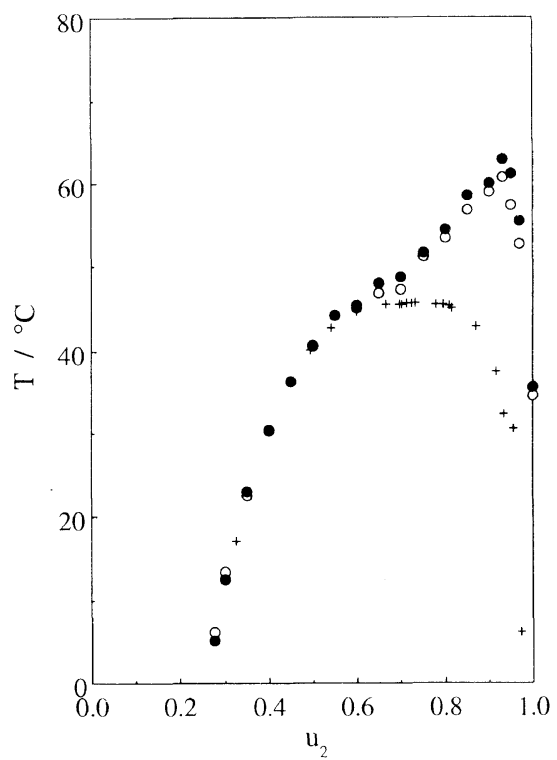

Figure 1. Coexistence curves of methanol/cyclohexane and methanol/cyclohexane/PVME systems: + , methanol/ cyclohexane; $\bigcirc$, methanol/cyclohexane/PVME $(c=0.003$ $\left.\mathrm{g} \mathrm{cm}^{-3}\right) ;$, methanol/cyclohexane/PVME $\left(c=0.01 \mathrm{~g} \mathrm{~cm}^{-3}\right)$ 
$u_{2}$ was found to be 0.71 , while the maximum demixing temperature of methanol/cyclohexane/PVME system was seen at $u_{2}=0.93$. The critical temperature of methanol/cyclohexane is $45.6^{\circ} \mathrm{C}$, while the demixing temperature at $u_{2}=0.93$ was $60.8^{\circ} \mathrm{C}$ for $c=0.003 \mathrm{~g} \mathrm{~cm}^{-3}$ and it shifted to $63.0^{\circ} \mathrm{C}$ for $c=0.01 \mathrm{~g} \mathrm{~cm}^{-3}$. In the region of $u_{2}$ higher than 0.6 , the demixing temperature increased with increasing the concentration of PVME from $c=0.003 \mathrm{~g} \mathrm{~cm}^{-3}$ to $0.01 \mathrm{~g} \mathrm{~cm}^{-3}$.

\section{SANS AND SAXS MEASUREMENTS}

\section{Principle}

For the purpose of determining the binary cluster integrals we consider semidilute polymer solutions in the poor solvent regime the condition of which was discussed in detail previously. ${ }^{7}$ The angular dependence of the intensity $I(q)$ of the scattered neutrons and $\mathrm{X}$-rays from a semidilute solution at polymer concentration $c$ is given by the following equations ${ }^{8}$ in the (so-called) intermediate range in which scattering wave vector $q$ lies between the reciprocal of the radius of gyration of a polymer chain and that of the size of a polymer segment:

$$
I(q)^{-1}=I(0)^{-1}\left(1+\xi^{2} q^{2}\right)
$$

where the correlation length $\xi$ is given by

$$
\begin{aligned}
& \left(1 / \xi^{2}-6 / M_{n} A^{2}\right) / c \\
& \quad=12 N_{\mathrm{A}} B_{1} / A^{2}+36 N_{\mathrm{A}}^{2} B_{2} c / A^{2}
\end{aligned}
$$

In the above equation, $M_{n}$ is the number average molecular weight of the polymer, $\left(M_{n} A^{2} / 6\right)^{1 / 2}$ is the unperturbed gyration radius of the polymer molecule, $N_{\mathrm{A}}$ the Avogadro number, $B_{1}$ and $B_{2}$ the binary and ternary cluster integrals ${ }^{7}$, respectively. The value $A$ is estimated as $0.9 \AA$ from the literature. ${ }^{9}$ By measuring the concentration dependence of $\xi$ at a temperature we are able to determine the values of cluster integrals at that temperature.

\section{SANS Measurements}

The SANS experiments were performed with the KENS-SAN instrument at KEK (National Laboratory of High Energy Physics at Tsukuba).

The instrument is installed on a pulsed cold neutron source and all wavelengths between 3.0 and $8.3 \AA$ were used at the same time for the measurement to increase the counting efficiency. The detector used was a two dimensional position sensitive detector (2DPSD) composed of 43 one-dimensional position sensitive counters and was set at a distance $3 \mathrm{~m}$ from the sample. The direct beam was focused on the center of the detector by using a converging Soller slit. The intensity of the incident beam was $4 \times 10^{4} \mathrm{n} \mathrm{s}^{-1} \mathrm{~cm}^{-2}$ and the area of the cross section of the beam at the sample was $14 \times 35 \mathrm{~mm}^{2}$. The sample cell was made of fused quartz and the thickness of the sample solution was $2 \mathrm{~mm}$.

The sample solutions consist of (hydrogenated) PVME dissolved in deuterated methanol, the characteristics of which are summarized in Table I. Deuterated methanol ( 99.8 atom \% deuterated) was purchased from Aldrich Chemical Company Inc.

The excess scattering intensity of methanol/PVME compared with that of methanol was determined after the transmission correction was made both for solution and solvent. In order to compensate the uniform incoherent scattering due to the hydrogen atoms we have added normal methanol into the solvent so that the solvent and sample solution have the same number of protons. The incident neutron spectrum as well as the detector efficiency of 2D-PSD was corrected for using the data of

Table I. Characteristics of the the PVME solutions in deuterated methanol used for SANS measurements

$\begin{array}{ll}M_{n} / \mathrm{g} \mathrm{mol}^{-1} & 4.65 \times 10^{4}\left(M_{w} / M_{n} \leqq 2.12\right) \\ c / \mathrm{g} \mathrm{cm}^{-3} & 0.0501,0.0647,0.0783,0.0930 \\ & 0.1068,0.1205,0.1344 \\ T /{ }^{\circ} \mathrm{C} & 40.0\end{array}$




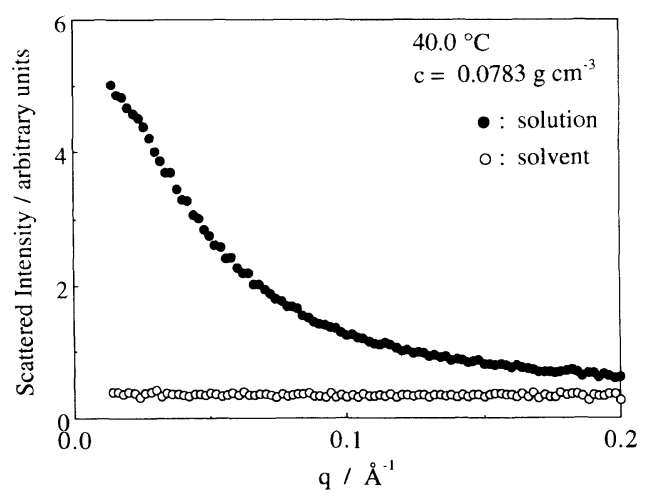

(a)

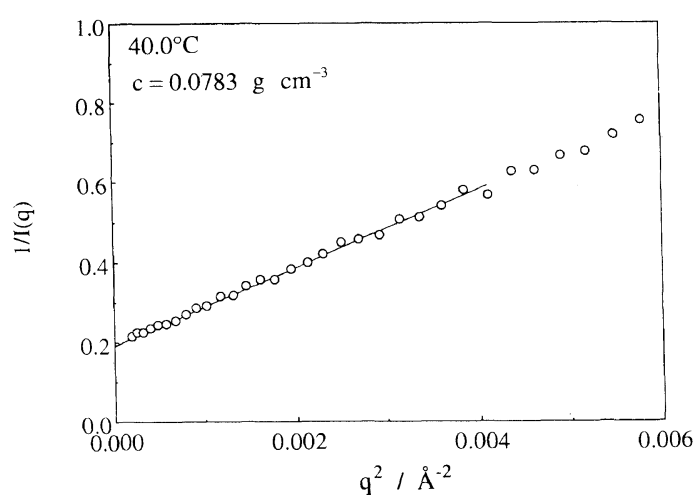

(b)

Figure 2. Examples of the angular dependences of SANS: (a) SANS from semidilute solution of PVME in deutrated methanol and that from deutrated methanol (the solvent); , solution; $\bigcirc$, solvent; (b) Lorentzian plot of the excess scattering from solution.

incoherent scattering from water.

Figure $2 \mathrm{a}$ shows an example of the angular dependences of the scattered neutron from the solution and the solvent; the latter is independent of $q$ in the measured small angle range. In Figure $2 b$ the reciprocal of $I(q)$, the intensity of the excess scattering from the solution over the solvent, is plotted against $q^{2}$, which demonstrate that the measured $I(q)$ obeys eq 1 in the range $q^{2}<4 \times 10^{-3} \AA^{-2}$ and we could determine $\xi$ for each concentration. In order to judge whether methanol is a good solvent or not we plotted $\log \xi$ against $\log c$ as shown in Figure 3. By now it has been established that semidilute polymer solutions

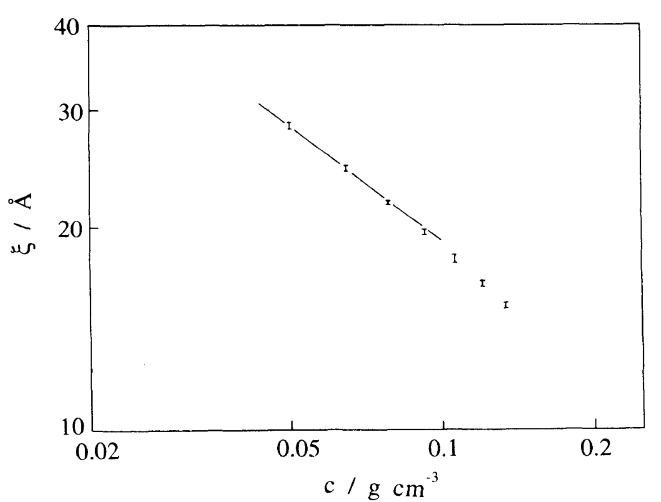

Figure 3. Concentration dependence of the correlation length $\xi$ of PVME in deuterated methanol at $40^{\circ} \mathrm{C}$. The slope of the line is -0.57 .

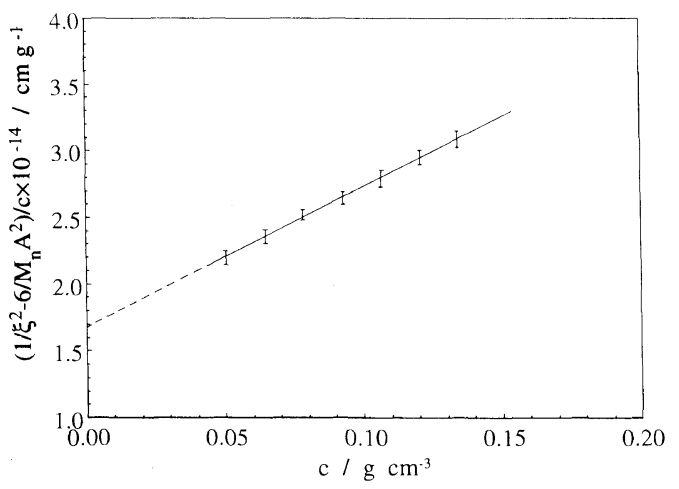

Figure 4. Concentration dependence of the correlation length $\xi$ of PVME in deuterated methanol at $40^{\circ} \mathrm{C}$ plotted according to eq 2 .

are classified into three regimes: good, marginal and theta, in the order of increasing concentration. ${ }^{10}$ The marginal and theta regimes together constitute the poor solvent regime where eq 1 and 2 hold. ${ }^{7}$ In the $\log \xi$ versus $\log c$ plot, the crossover from the good regime to the marginal one is characterized by a change in the slope from -0.75 to -0.5 , whereas the crossover in the poor solvent regime, i.e., the crossover from marginal regime to theta regime, the slope must change from -0.5 to -1 . Figure 3 shows that the slope of the line is about -0.57 in the lower concentration region and the slope becomes steeper in the higher concentration region. We thus conclude 
Table II. $\mathrm{B}_{1}$ and $\mathrm{B}_{2}$ of PVME in deuterated methanol at $40^{\circ} \mathrm{C}$ deduced from

SANS measurements

$\begin{array}{ll}B_{1} / \mathrm{cm}^{3} & 1.88 \times 10^{-27} \\ B_{2} / \mathrm{cm}^{6} & 6.53 \times 10^{-51}\end{array}$

Table III. Characteristics of the PVME solutions in cyclohexane used for SAXS measurements

$M_{n} / \mathrm{g} \mathrm{mol}^{-1} \quad 4.65 \times 10^{4}\left(M_{w} / M_{n} \leqq 2.12\right)$

$c / \mathrm{g} \mathrm{cm}^{-3} \quad 0.0748,0.0978,0.1302,0.1400,0.1605$

$T /{ }^{\circ} \mathrm{C} \quad 38.5,39.4,40.6,41.8,42.9,45.2,47.4$

that PVME in methanol is in the poor solvent regime at this temperature. The concentration dependence of $\xi$ for methanol/PVME determined from eq 1 was found to obey eq 2 as shown in Figure 4. Thus we were able to determine $B_{1}$ and $B_{2}$ separately as listed in Table II.

\section{SAXS Measurements}

The SAXS measurements were carried out by using a Kratky U-slit camera with entrance slit of $100 \mu \mathrm{m}$, counter slit of $300 \mu \mathrm{m}$, and height-determining slit of $16 \mathrm{~mm}$. A RigakuDenki RU-200 rotating copper anode X-ray generator was used at $50 \mathrm{kV}, 200 \mathrm{~mA}$ and a wavelength $\lambda=1.54 \AA$ was selected with $\mathrm{Ni}$ filter of $14 \mu \mathrm{m}$ thickness. Photon counting was done by a scintillation counter with pulse height analyzer. Slit correction was done by the variation-function method. ${ }^{11}$ The thickness of the sample cell used was $2 \mathrm{~mm}$. The temperature of the sample solution was controlled within $\pm 0.03^{\circ} \mathrm{C}$ by using a thermocouple placed in the solution. The characteristics of the sample solutions are summarized in Table III.

The measured scattered intensity of cyclohexane/PVME obeyed eq 1 in the range $q^{2}<4 \times 10^{-3} \AA^{-2}$ as shown in Figure 5. From this figure we could determine the correlation length $\xi$ for each concentration and temperature. The concentration dependence of $\xi$ for

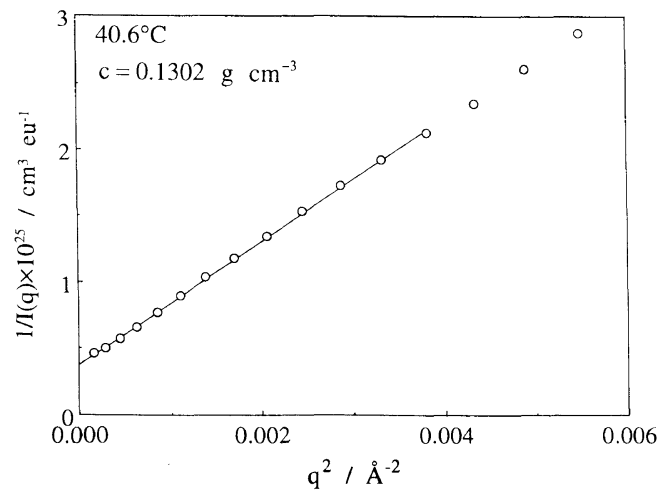

Figure 5. An example of the angular dependence of SAXS from semidilute solution of PVME in cyclohexane.

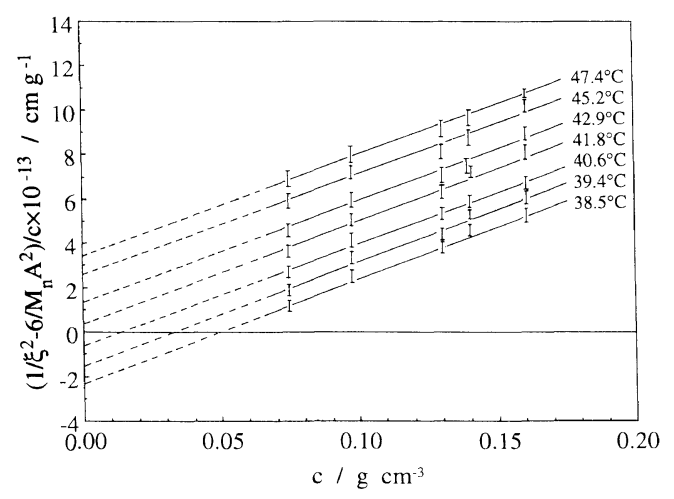

Figure 6. Concentration dependence of the correlation length $\xi$ of PVME in cyclohexane at several temperature near the $\theta$ point plotted according to eq 2 .

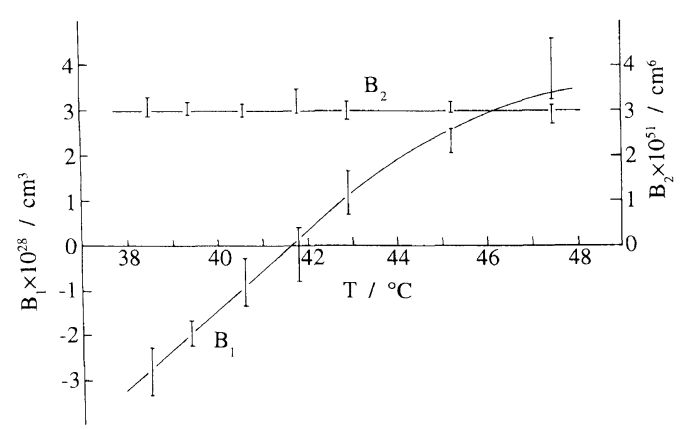

Figure 7. Temperature dependences of binary $\left(B_{1}\right)$ and ternary $\left(B_{2}\right)$ cluster integrals of PVME in cyclohexane.

each temperature turned out to agree well with the prediction of eq 2 as shown in Figure 6. Thus we were able to determine $B_{1}$ and $B_{2}$ separately as a function of temperature. Figure 
7 shows the temperature dependence of $B_{1}$ and $B_{2}$ thus obtained. We see from this figure that temperature at which $B_{1}$ vanishes, $\theta$, is about $41.6^{\circ} \mathrm{C}$.

\section{SPINODAL CALCULATION}

As described in the section of MEASUREMENT OF COEXISTENCE CURVE the phase separation behavior of the ternary system at constant (small) polymer concentration has a characteristic feature that, in the region of solvent composition from pure methanol to the region near the critical demixing point, the coexistence curve of the ternary system essentially coincides with the binodal curve of the solvent mixture, whereas upon increasing the fraction of cyclohexane from this region on, the coexistence curve of the ternary system begins to deviate significantly from the binodal curve of the solvent mixture. In the following we analyze this behavior theoretically.

In view of the polydispersity of the PVME samples we must be contented with a qualitative analysis. For this purpose an approach of spinodal calculation based on the FloryHuggins lattice model adopted by some authors $^{12}$ is quite convenient. The free energy of mixing (per lattice site) of the ternary system reads

$$
\begin{aligned}
\Delta G / k T= & \phi_{1} \ln \phi_{1}+\phi_{2} \ln \phi_{2}+(1 / N) \phi_{3} \ln \phi_{3} \\
& +\chi_{12} \phi_{1} \phi_{2}+\chi_{13} \phi_{1} \phi_{3}+\chi_{23} \phi_{2} \phi_{3}
\end{aligned}
$$

where $\phi_{i}(i=1,2,3)$ is the volume fraction of component $i, N$ the degree of polymerization of PVME, $\chi_{i j}$ the interaction parameter between components $i$ and $j$. Again we denote methanol as component 1 , cyclohexane as component 2, and PVME as component 3. Applying the spinodal condition:

$$
\begin{array}{r}
\left(\partial^{2} \Delta G / \partial \phi_{2}{ }^{2}\right)\left(\partial^{2} \Delta G / \partial \phi_{3}{ }^{2}\right) \\
-\left(\partial^{2} \Delta G / \partial \phi_{2} \partial \phi_{3}\right)^{2}=0
\end{array}
$$

to the above free energy we obtain the following equation for the spinodal.

$$
\begin{aligned}
\phi_{1} & +\phi_{2}+N \phi_{3}-2\left(\chi_{1}+\chi_{2}\right) \phi_{1} \phi_{2} \\
& -2 N\left(\chi_{1}+\chi_{3}\right) \phi_{1} \phi_{3}-2 N\left(\chi_{2}+\chi_{3}\right) \phi_{2} \phi_{3} \\
& +4 N\left(\chi_{1} \chi_{2}+\chi_{1} \chi_{3}+\chi_{2} \chi_{3}\right) \phi_{1} \phi_{2} \phi_{3}=0
\end{aligned}
$$

where

$$
\begin{aligned}
& 2 \chi_{1}=\chi_{12}+\chi_{13}-\chi_{23} \\
& 2 \chi_{2}=\chi_{12}+\chi_{23}-\chi_{13} \\
& 2 \chi_{3}=\chi_{13}+\chi_{23}-\chi_{12}
\end{aligned}
$$

We have calculated the spinodal curve by solving eq 5 for each composition of the solvent mixture at constant polymer concentration $\left(\phi_{3}\right)$ assuming that each "enthalpy parameter" $\chi_{i j} T$ is independent of temperature and concentration.

The value of the parameter $\chi_{12} T$ has been determined as in the following. The lattice model gives $\chi_{12}=2$ at the critical demixing point of the solvent mixture, and the experimentally observed critical point occurs at $45.6^{\circ} \mathrm{C}$, so we have $\chi_{12} T=638$.

We have calculated the values of $\chi_{13} T$ and $\chi_{23} T$ from the respective binary cluster integrals deduced from the small angle scattering experiments described in the preceding section. The lattice model gives the following relationship between $\chi$-parameter and the binary cluster integral $B_{1}$,

$$
\chi_{i j}=(1 / 2)\left[1-\left(v_{i} / \bar{v}_{3}{ }^{2}\right) N_{\mathrm{A}} B_{1}\right]
$$

where $\bar{v}_{3}$ is the specific volume of the polymer, and $v_{i}$ is the molar volume of either methanol $(i=1)$ or cyclohexane $(i=2)$.

As for methanol/PVME system there arises a delicate problem concerning the association among methanol molecules by hydrogen bonding: In order to determine $v_{1}$ we need to know how many methanol molecules are linked together by hydrogen bonding. There exist a number of experimental' and theoretical work of alcohol structure ${ }^{13-15}$. Despite some discrepancies among the published data the presence of the predominant amount of $n$-mers $(n \geqq 4)$ seems to be a well established fact. We therefore calculated the value of $\chi_{13} T$ by 


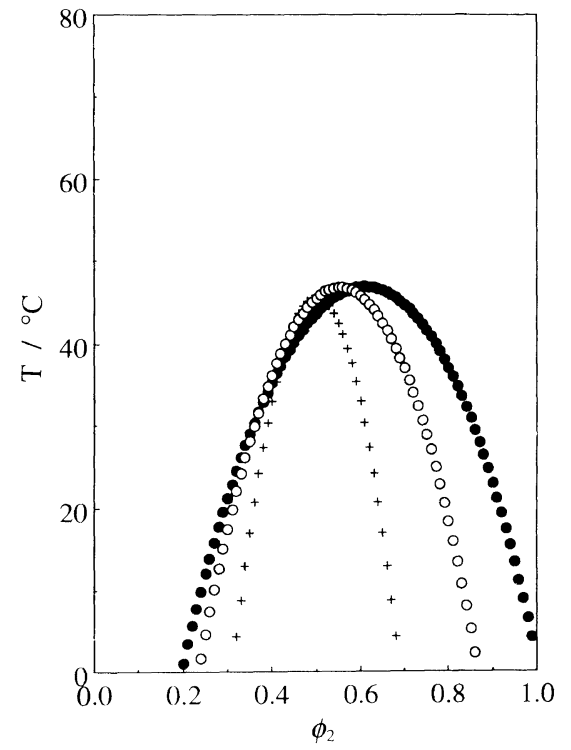

Figure 8. Spinodal curves of the ternary system methanol/cyclohexane/PVME: association number of methanol molecules $n=4$; + , methanol/cyclohexane; $\bigcirc$, methanol/cyclohexane/PVME $\left(\phi_{3}=0.003\right)$;, methanol/cyclohexane/PVME $\left(\phi_{3}=0.01\right)$.

tentatively assuming $n=4, n=6$, and $n=10$ for $v_{i}$, together with the experimentally determined values of $B_{1}=1.9 \times 10^{-27} \mathrm{~cm}^{3}$ at $40^{\circ} \mathrm{C}$, and $\bar{v}_{3}=0.869 \mathrm{~cm}^{3} \mathrm{~g}^{-1}$ at $30^{\circ} \mathrm{C}$. The results are $\chi_{13} T=119.0$ for $n=4\left(v_{1}=160 \mathrm{~cm}^{3}\right.$, caluculated from the literature value of density $\left.0.791 \mathrm{~g} \mathrm{~cm}^{-316}\right), \chi_{13} T=100.2$ for $n=6\left(v_{1}=\right.$ $\left.240 \mathrm{~cm}^{3}\right)$, and $\chi_{13} T=62.7$ for $n=10\left(v_{1}=\right.$ $400 \mathrm{~cm}^{3}$ ).

The parameter $\chi_{23} T$ for cyclohexane/PVME system has been determined by measuring the temperature $\theta$ at which $B_{1}$ vanishes. We found $\theta=41.6^{\circ} \mathrm{C}$, which gives $\chi_{23} T=157$.

Spinodal curves of the ternary system at constant polymer concentration thus calculated are shown in Figure 8 for $n=4$, Figure 9 for $n=6$, and Figure 10 for $n=10$. Comparing these curves with the experimental curve Figure 1 we see that in the case $n \gtrsim 6$ our spinodal calculation reproduces at least qualitatively well the essential feature of the experimental phase separation behavior. Thus our calculation seems to support that average association

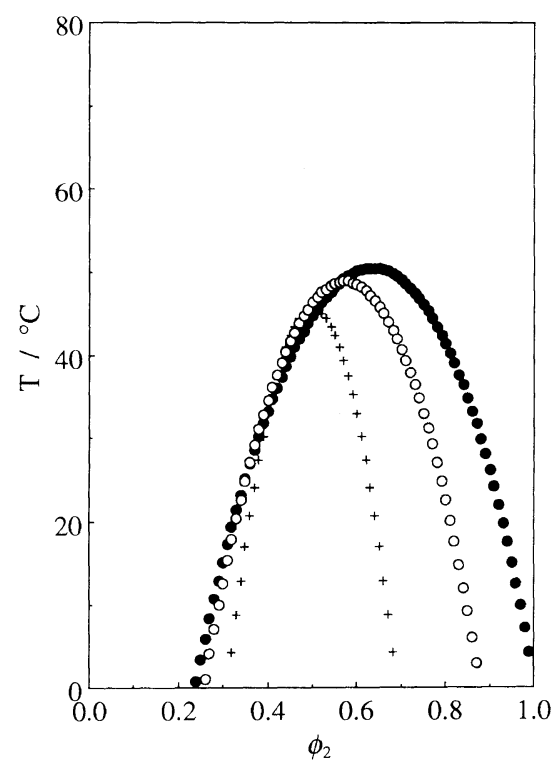

Figure 9. Spinodal curves of the ternary system methanol/cyclohexane/PVME: association number of methanol molecules $n=6$; + , methanol/cyclohexane; $\bigcirc$, methanol/cyclohexane/PVME $\left(\phi_{3}=0.003\right) ; 0$, methanol/cyclohexane/PVME $\left(\phi_{3}=0.01\right)$.

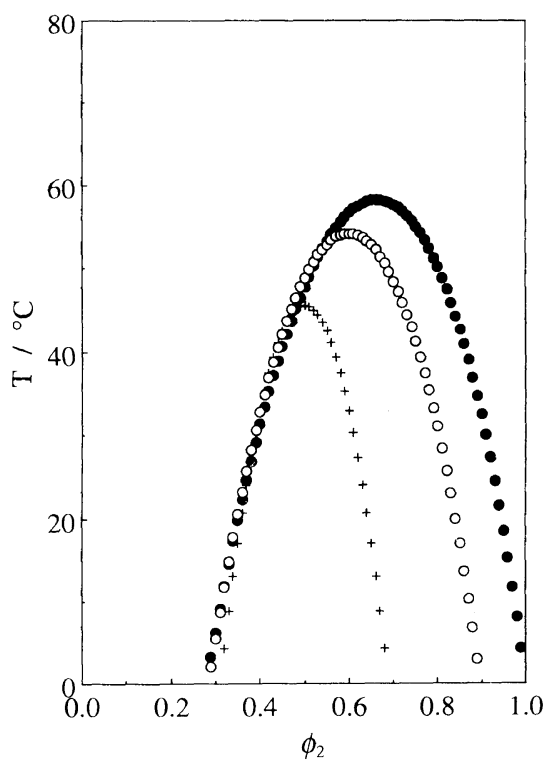

Figure 10. Spinodal curves of the ternary system methanol/cyclohexane/PVME: association number of methanol molecules $n=10 ;+$, methanol/cyclohexane; $\bigcirc$, methanol/cyclohexane/PVME $\left(\phi_{3}=0.003\right)$;, methanol/cyclohexane/PVME $\left(\phi_{3}=0.01\right)$. 
number $n$ of methanol molecules is about 6 or even larger. Finally seemingly anomalous behavior of the observed binodal curve in the cyclohexane-rich region remains to be explained. This problem is, however, can not be clarified until the binodal curve itself is calculated by taking the polydispersity in both PVME and the association of methanol molecules into account, which is outside the scope of the present paper.

\section{CONCLUSION}

The coexistence curve of the ternary solution made up of methanol, cyclohexane, and a constant (small) amount of PVME exhibits a notable feature: the presence of small amount of PVME does not appreciably disturb the coexistence curve of binary methanol/cyclohexane system in the methanol-rich region, whereas in the cyclohexane-rich region it causes a sizable shift of phase boundary toward higher temperatures.

Invoking the recognized similarity of binodal and spinodal curves, by calculating the spinodal curves based on Flory-Huggins solution theory, we were able to explain the above mentioned phase behavior as due to the smallness of $\chi_{13}$ parameter.

The smallness of $\chi_{13}$ can be reconciled with the value of binary cluster integral deduced from SANS experiment only if, in conformity with the current knowledge of the structure of alcohols, we admit that methanol molecules form rather large clusters due to hydrogen- bonding.

Acknowledgments. The present authors express their sincere thanks to Dr. Eiichi Wada for the guidance to X-ray and neutron small angle experiments and the critical reading of the manuscript. They also would like to thank Prof. Satoru Mashimo for valuable discussions on the association of alcohol molecules.

\section{REFERENCES}

1. P. G. de Gennes, J. Phys. (Paris), 37, L59 (1976).

2. Y. Izumi, A. Dondos, C. Picot, and H. Benoit, Makromol. Chem., 180, 2483 (1979).

3. A. Dondos and Y. Izumi, Makromol. Chem., 181, 701 (1980).

4. G. Staikos, P. Skondras, and A. Dondos, Makromol. Chem., 183, 603 (1982).

5. F. Tanaka and H. Ushiki, Macromolecules, 21, 1041 (1988).

6. A. Seidell, "Solubilities of Organic Compounds," D. Van Nostrand, New York, N.Y., 1941, p 53.

7. K. Okano, T. Ichimura, K. Kurita, and E. Wada, Polymer, 28, 693 (1987).

8. M. A. Moore, J. Phys. (Paris), 38, 265 (1977).

9. B. J. Bauer, B. Hanley, and Y. Muroga, Polym. Commun., 30, 19 (1989).

10. D. W. Schaefer, Polymer, 25, 387 (1984).

11. O. Glatter, J. Appl. Crystallogr., 7, 147 (1974).

12. H. G. Schild, M. Muthukumar, and D. A. Tirrell, Macromolecules, 24, 948 (1991).

13. W. M. Bartczak, Ber. Bunsenges. Phys. Chem., 83, 987 (1979).

14. S. Mashimo, N. Miura, T. Umehara, S. Yagihara, and K. Higasi, J. Chem. Phys., 96, 6358 (1992).

15. W. A. P. Luck, Discuss. Faraday Soc., 43, 1157 (1967).

16. "International Critical Tables," Vol. III, McGraw Hill, New York, N.Y., 1928, p 27. 